\title{
Optical Investigation of Quantum Confinement in PbSe Nanocrystals at Different Points in the Brillouin Zone
}

\author{
Rolf Koole, Guy Allan, Christophe Delerue, Andries Meijerink,* \\ Daniël Vanmaekelbergh, and Arjan J. Houtepen*
}

$W_{e}$ present detailed investigations on the optical properties of PbSe nanocrystals. The absorption spectra of monodisperse, quasispherical nanocrystals exhibit sharp features as a result of distinct optical transitions. To study the size dependence, absorption spectra of nanocrystals ranging from 3.4 to $10.9 \mathrm{~nm}$ in diameter are analysed and a total of 11 distinct optical transitions are identified. The assignment of the various optical transitions is discussed and compared to theoretically calculated

\section{Keywords:}

- chalcogenides

- multiple exciton generation

- quantum dots

- size-dependent properties

- UV/Vis spectroscopy
[*] R. Koole, Prof. A. Meijerink, Prof D. Vanmaekelbergh,

Dr. A. J. Houtepen ${ }^{+}$

Condensed Matter and Interfaces, University Utrecht

POB 80000, 3508 TA Utrecht (The Netherlands)

Fax: $(+31)$ 30-2532403

E-mail: a.meijerink@phys.uu.nl

a.j.houtepen@tudelft.nl

Dr. G. Allan, Prof. C. Delerue

Institut d'Electronique, de Microélectronique et

de Nanotechnologie (UMR CNRS 8520)

Département ISEN

41 boulevard Vauban, 59046 Lille Cedex (France)

$\left.{ }^{+}\right]$Current address:

Faculty of Applied Sciences

Delft University of Technology

Julianalaan 136

2628 BL Delft (The Netherlands)

Supporting Information is available on the WWW under http:// www.small-journal.com or from the author.

\section{Introduction}

In recent years the interest in lead chalcogenide colloidal nanocrystals (NCs) has grown considerably. The bulk materials are interesting as they are IV-VI compounds with a $\mathrm{Pb}$ atomic configuration $[\mathrm{Xe}] 4 \mathrm{f}^{14} 5 \mathrm{~d}^{10} 6 \mathrm{~s}^{2} 6 \mathrm{p}^{2}$. As a consequence of the high atomic number of $\mathrm{Pb}$, and the resulting high electron velocities, relativistic effects localize the 6 s orbital, transforming $\mathrm{Pb}$ into a $6 \mathrm{p}^{2}$ atom (inert pair effect). ${ }^{[1]}$ Another consequence of the high atomic number of $\mathrm{Pb}$ is the relatively large ratio of ionic radii $\left(r_{\mathrm{Pb}, 2+} / r_{\mathrm{Se}, 2-}=0.59\right)$. As a result, the coordination number of $\mathrm{PbSe}(6)$ is higher than for, for example, CdSe (4). The PbSe rock-salt lattice leads to an electronic band structure that is different from that of the typical II-VI compounds. An important consequence is that the valence-band maximum (VBM) and conduction-band minimum (CBM) are both situated at the L- 
point in the Brillouin zone (see Figure 1), which is fourfold degenerate (eightfold including spin degeneracy).

As a result of the small effective mass $(m)$ of the charge carriers at the bottom of the valence band and top of the

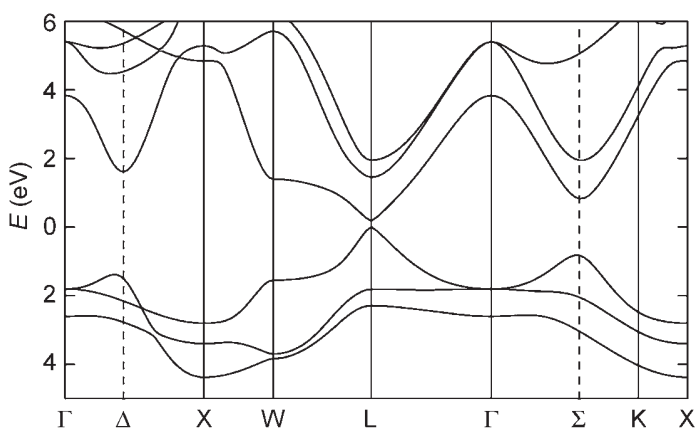

Figure 1. Band structure of bulk PbSe. The zero of energy is located at the top of the valence band. ${ }^{[1]}$

conduction band $\left(m_{\mathrm{h}}^{*} \approx m_{\mathrm{e}}^{*}=0.1\right.$, where subscripts $\mathrm{h}$ and $\mathrm{e}$ stand for holes and electrons, respectively) and the large bulk exciton Bohr radius of $46 \mathrm{~nm}$, the effect of quantum confinement is particularly strong in PbSe. ${ }^{[2,3]}$ Since the room-temperature bandgap of the bulk material is only $0.278 \mathrm{eV}$, it is possible to tune the $\mathrm{NC}$ bandgap from $0.278 \mathrm{eV}$ (ca. $4400 \mathrm{~nm}$ ) to over $1.1 \mathrm{eV}$ (ca. $1100 \mathrm{~nm}$ ) by changing the size of the nanocrystal. Therefore, lead chalcogenide quantum dots are promising building blocks for a wide number of optoelectronic applications in the near infrared. ${ }^{[3-6]}$ For example, PbSe QDs hold great promise in the field of photovoltaics because of the recently observed multiple exciton generation (MEG) in these nanocrystals. ${ }^{[7-10]}$

Here we report on detailed investigations of the optical absorption spectra of PbSe QDs. First, the assignment of the multiple features (up to 11) in the absorption spectrum of highly monodisperse $\mathrm{PbSe}$ QDs is discussed and compared to calculated spectra of $\mathrm{PbSe}$ QDs that were recently published. ${ }^{[1,11]}$ Next, the size dependence of the energy of the various transitions is analyzed for 16 different sizes of $\mathrm{PbSe}$ QDs, ranging from 3.4 to $10.9 \mathrm{~nm}$. It follows that the higher-energy transitions can be assigned to optical transitions at a different point in the Brillouin zone than the lower-energy transitions. We first use effective mass theory to qualitatively explain the observed differences in the optical transitions.
Subsequently, we apply tight-binding calculations to quantitatively confirm the assignment of the different transitions to different points in the Brillouin zone. Finally, it is discussed how these observations may be relevant for the explanation of MEG, the mechanism of which is still under debate.

\section{Results and Discussion}

The absorption $(A)$ spectrum of a dispersion of nanocrystals with a diameter of $6.8 \pm 0.3 \mathrm{~nm}$, determined by transmission electron microscopy (TEM) measurements, is shown in Figure 2A and B. The narrow size distribution results in sharp optical transition features: the full width at half-maximum of the lowest energy transition in Figure 2 is only $55 \mathrm{meV}$ and many features can be distinguished in the spectrum. The second derivative of the absorption allows us to identify the different optical transitions and the corresponding transition energies. ${ }^{[10]}$ This is shown in Figure $2 \mathrm{C}$ and D. A minimum in the second derivative corresponds to the maximum of an absorption band (note that the second derivative is plotted with the minima upwards). Altogether, 11 minima can be distinguished in Figure 2.

One has to be careful in assigning optical transitions based on the second derivative. Noise in the absorption spectrum can create peaks in $\mathrm{d} A^{2} / \mathrm{d}^{2} E$. Absorption peaks caused by vibrational overtones of, for example, $\mathrm{C}-\mathrm{H}$ or $\mathrm{C}=\mathrm{O}$ vibrations in capping molecules or reagents can also interfere. To avoid the influence of noise, we have only included features that were present in spectra of several nanocrystal batches. To avoid optical transitions from organic material, we have only included features that shift with par-
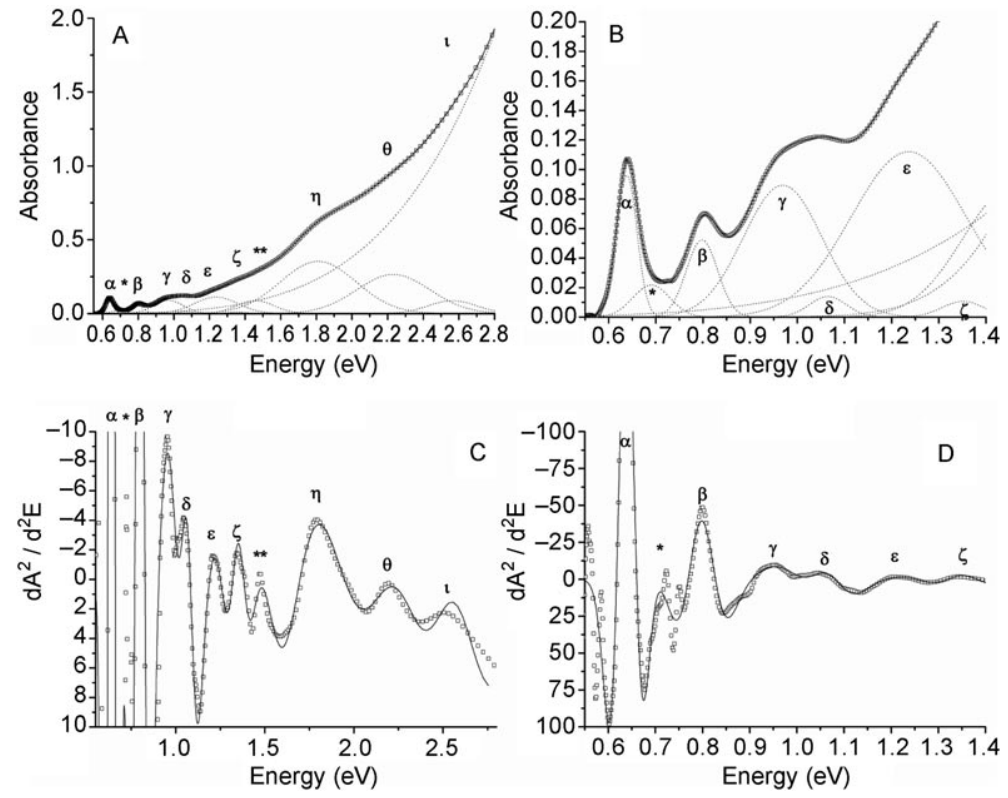

Figure 2. A) Optical absorption spectra of a dispersion of 6.8-nm quasi-spherical PbSe NCs in tetrachloroethylene. The open squares represent the experimental spectrum, the solid line is a fit to Gaussian components and a background that increases with $E^{4}$ (dotted lines). The low-energy part of the same spectrum and corresponding fit are shown in (B). The second derivatives of the experimental spectrum and the fit are shown in (C) and (D) as the open squares and solid lines, respectively. 
ticle size. Finally, one has to be aware that the second derivative itself can create artefacts. For example, the second derivative of two well-separated Gaussians has an additional minimum in between the two peaks. To analyze if the minima in the second derivative correspond to actual optical transitions, we have fitted the absorption spectrum in Figure 2A to multiple Gaussian functions. A background function that increases with $A \propto E^{4}$ was also included in the fit, corresponding to weak Rayleigh scattering of the nanocrystals. The multi-Gaussian fits of the absorption spectrum is plotted as a solid line in Figure 2A and B, and the 11 associated Gaussian functions and background function are shown as dotted lines. As can be seen in Figure 2A and B, there is excellent agreement between the multi-Gaussian fit and the absorption spectrum. The second derivative of the multiGaussian fit is shown as a solid line in Figure 2C and D and is in good agreement with the second derivative of the absorption spectrum. Importantly, we found that it is necessary to include all 11 Gaussians shown in Figure 2A to obtain all the features in the second derivative. We conclude that they all correspond to optical transitions.

The optical transitions are labelled with Greek letters. Transitions that, to our knowledge, have not previously been reported are labelled with asterisks. The assignment of the optical transitions is subject to considerable debate in the literature. ${ }^{[1,10-13]}$ This debate largely focuses on the assignment of the "second" peak in the absorption spectrum, labelled $\beta$ here. Based on theoretical calculations of the energy levels and experimental work, this feature has previously been assigned to the $1 \mathrm{~S}_{\mathrm{h}}-1 \mathrm{P}_{\mathrm{e}}$ (or $1 \mathrm{P}_{\mathrm{h}}-1 \mathrm{~S}_{\mathrm{e}}$ ) transition. ${ }^{[11,14]}$ However, these transitions are optically forbidden and are expected to be very weak. Liljeroth et al. were able to measure the density of states of single PbSe nanocrystals directly using scanning tunnelling spectroscopy and suggested that transition $\beta$ corresponds to the $1 \mathrm{P}_{\mathrm{h}}-1 \mathrm{P}_{\mathrm{e}}$ transition. ${ }^{[13]}$ Below we will compare the experimental absorption spectra and their features with an absorption spectrum that we obtained by tight-binding calculations, as well as an absorption spectrum that was recently calculated by An et al. ${ }^{[1]}$ As will become clear, there are still important discrepancies between the experimental data and both theoretical calculations. The purpose of this comparison is to give an overview of the current understanding of the absorption spectrum of $\mathrm{PbSe} \mathrm{QDs}$, not to give a final answer on the correct assignment of the different transitions.

Using tight-binding calculations as reported earlier, the absorption spectrum of a $\mathrm{PbSe} \mathrm{QD}$ of $6.1 \mathrm{~nm}$ in diameter was calculated. ${ }^{[1]}$ The calculated spectrum is plotted together with the experimental spectrum of PbSe QDs with a diameter of $6.8 \mathrm{~nm}$ (see Figure 3). In energy, this is the best matching experimental spectrum that we have measured compared to the calculated spectrum. The second derivative of the experimental spectrum is shown as well to identify the position of the different transitions more precisely. The first transition $(\alpha)$ of the calculated spectrum $\left(a 1 S_{h}-1 S_{e}\right.$ transition) is slightly blue-shifted with respect to the experimental spectrum, which is consistent with the smaller nanocrystal size. Transition $\beta$ is not reproduced by the calculations because the $1 \mathrm{~S}_{\mathrm{h}}-1 \mathrm{P}_{\mathrm{e}}$ (or $1 \mathrm{P}_{\mathrm{h}}-1 \mathrm{~S}_{\mathrm{e}}$ ) transition that is cal-

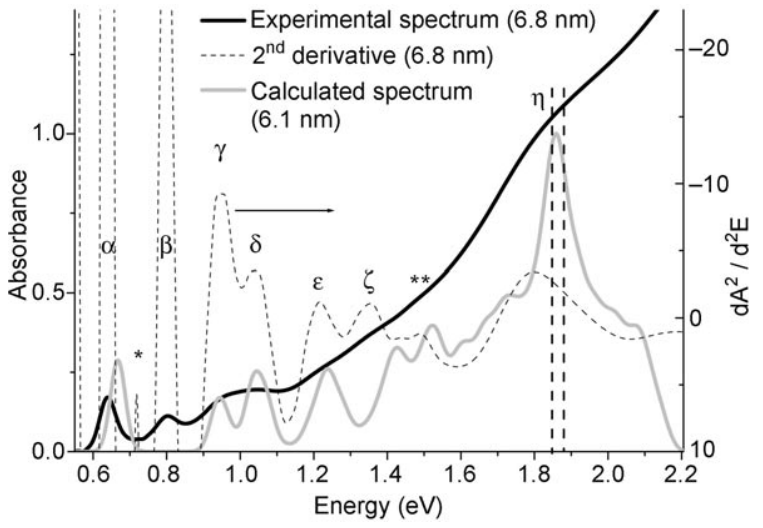

Figure 3. Experimental absorption spectrum of $\mathrm{PbSe} \mathrm{QDs}$ with diameter $6.8 \pm 0.3 \mathrm{~nm}$ (solid black line). For clarity, the second derivative of the experimental spectrum is plotted on a different scale (right $y$-axis). The absorption spectrum obtained by tight-binding calculations of a PbSe QD with a diameter of $6.1 \mathrm{~nm}$ is shown in gray, scaled to an appropriate value for comparison with the experimental spectrum. The heavy-gray dashed vertical lines indicate the energy range that was used in the tight-binding calculations to determine from which points in the Brillouin zone these transitions originate. The decrease of the theoretical spectrum to zero above $2 \mathrm{eV}$ is due to the limited number of eigenstates that we can calculate (here 1100 states).

culated for this energy has no oscillator strength. Nevertheless, transitions $\gamma$ and $\delta$ are reproduced and have a $1 \mathrm{P}_{\mathrm{h}}-1 \mathrm{P}_{\mathrm{e}}$ character, according to the tight-binding calculations. Also transition $\varepsilon$ is calculated, and transitions $\zeta$ and $* *$ show up in the theoretical spectrum as well (although slightly shifted). In addition, the relative oscillator strengths calculated for all these transitions are in good agreement with experiment. The tight-binding calculations also reproduce transition $\eta$ at approximately $1.85 \mathrm{eV}$ with large oscillator strength. In summary, the tight-binding calculations reproduce the measured absorption spectrum of a nanocrystal with similar size, except for transition $\beta$, which, according to these calculations, is the forbidden $1 \mathrm{~S}_{\mathrm{h}}-1 \mathrm{P}_{\mathrm{e}}\left(\right.$ or $1 \mathrm{P}_{\mathrm{h}}-1 \mathrm{~S}_{\mathrm{e}}$ ) transition.

A different approach for the assignment of the different transitions was recently reported by $\mathrm{An}$ et al. ${ }^{[1]}$ Using pseudo-potential calculations, it was calculated that a $\mathrm{PbSe}$ nanocrystal with a $1 \mathrm{~S}_{\mathrm{h}}-1 \mathrm{~S}_{\mathrm{e}}$ transition $(\alpha)$ at $0.89 \mathrm{eV}$ has a $1 \mathrm{P}_{\mathrm{h}}-1 \mathrm{P}_{\mathrm{e}}$ transition $(\beta)$ that is $0.24 \mathrm{eV}$ higher in energy, at $1.13 \mathrm{eV}$. We have measured and analyzed the absorption spectrum of $\mathrm{PbSe}$ QDs that have a first transition $(\alpha)$ at $0.87 \mathrm{eV}$ (see Supporting Information, Figure S1), allowing for a good comparison with the calculated spectrum by An et al. We find that PbSe QDs with transition $\alpha$ at $0.87 \mathrm{eV}$ display a second transition $(\beta)$ at $1.12 \mathrm{eV}$, which coincides nicely with the values calculated by An et al. ${ }^{[1]}$ This suggests that the second peak in the absorption spectra of PbSe QDs is the $1 \mathrm{P}_{\mathrm{h}}-1 \mathrm{P}_{\mathrm{e}}$ transition, in line with the assignment by Liljeroth et al. ${ }^{[13]}$ but in contrast with the tight-binding calculations discussed above. Interestingly, An et al. calculate two additional transitions as a result of heavily mixed $\mathrm{P}$ - and D-like states, located at $1.38 \mathrm{eV}$ and approximately $1.48 \mathrm{eV}$, respectively. The energies corresponding to transitions $\gamma$ and 
$\delta$ in the measured absorption spectrum are 1.39 and $1.46 \mathrm{eV}$, respectively (see Supporting Information, Figure S1), in good agreement with the calculated values of An et al. Furthermore, An et al. calculated a transition in between transitions $\beta$ and $\gamma$, originating from anisotropically asymmetric transitions with small oscillator strengths. We were not able to resolve this weak transition from the measured absorption spectra of PbSe QDs of various sizes. It is important to note that the calculated spectrum of An et al. is based on a nanocrystal with a diameter of $6.1 \mathrm{~nm}$, whereas the corresponding absorption spectrum was measured for PbSe QDs with a diameter of $4.7 \pm 0.4 \mathrm{~nm}$, based on the analysis of TEM images. In other words, there is a striking difference of $220 \mathrm{meV}$ between the calculated energy of the first transition $(\alpha)$ of a PbSe QD of $6.1 \mathrm{~nm}$ using tight-binding calculations $(0.67 \mathrm{eV})$ or pseudo-potential calculations $(0.89 \mathrm{eV})$. As can be derived from Figure 4 (discussed below), we find an experimental value of $0.70 \mathrm{eV}$ for the first transition of a $\mathrm{PbSe}$ QD of $6.1 \mathrm{~nm}$.

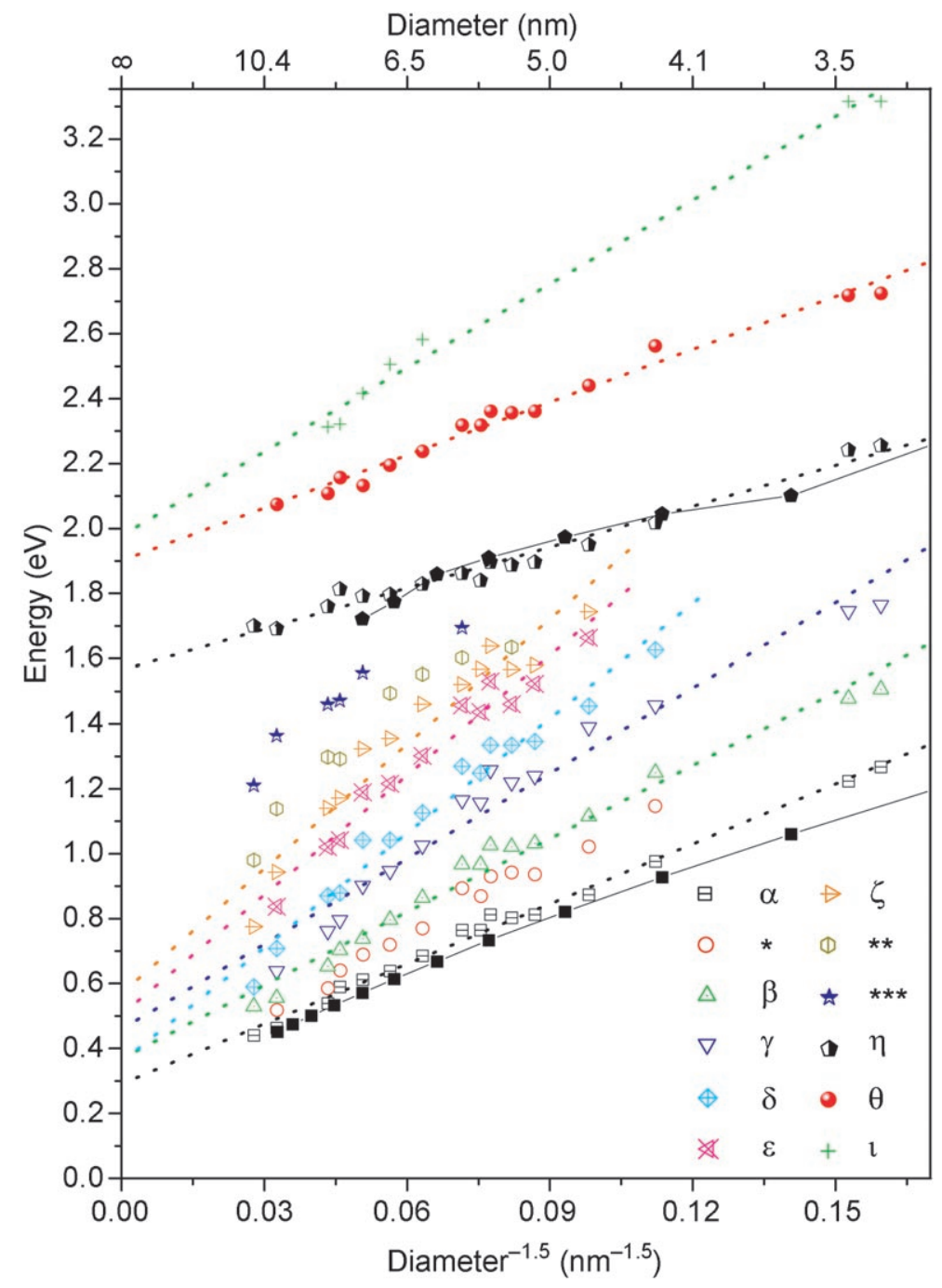

Figure 4. Energies of optical transitions as indicated in Figure 2 for PbSe nanocrystals of different sizes, ranging from 3.4 to $10.9 \mathrm{~nm}$ in diameter. The dotted lines are linear fits assuming a linear dependence between $E$ and $D^{-1.5}$, and serve as a guide for the eye. The solid black symbols and interconnecting solid black lines show the results of the size dependence using tight-binding calculations for transition a and the first transition at the $\Sigma$-point of the Brillouin zone, transition $\eta$.
Feature $*$ in Figure 2 is not clearly observed in the absorption spectra. However, it does show up in the secondderivative spectra we have studied (e.g., Figure 2D), and is situated in between transitions $\alpha$ and $\beta$. Several spectra from the literature also suggest an additional feature as a shoulder on the low-energy side of peak $\beta$. See, for instance, Figure 1 in the supporting information of ref. [15] or Figure 4 in ref. [5]. This feature may be the $1 \mathrm{~S}_{\mathrm{h}}-1 \mathrm{P}_{\mathrm{e}}\left(\right.$ or $1 \mathrm{P}_{\mathrm{h}}-$ $1 \mathrm{~S}_{\mathrm{e}}$ ) transition, implying that transition $\beta$ is the $1 \mathrm{P}_{\mathrm{h}}-1 \mathrm{P}_{\mathrm{e}}$ transition. More definitive proof of the existence of feature * as a real optical transition will require measurement of the absorption spectrum of a monodisperse sample at low temperature, or the use of excitation spectroscopy. ${ }^{[16]}$

To investigate the influence of quantum confinement on the various transitions, we have measured and analyzed the absorption spectra of quasispherical PbSe nanocrystals with diameters ranging from 3.4 to $10.9 \mathrm{~nm}$. The different transitions were assigned on the basis of the features in the second-derivative spectra, in a similar way as discussed for the spectrum in Figure 2. Additional absorption spectra and corresponding second-derivative spectra of various nanocrystal sizes can be found in the Supporting Information. Figure 4 shows the different transition energies of PbSe QDs as a function of the nanocrystal size. The $x$ axis has the dimension $D^{-1.5}$ because we found that the energy of the first transition in the spectra changes with the diameter $D$ as $E_{1 \mathrm{st}} \approx D^{-1.5}$. This is a somewhat stronger dependence on size than that reported by $\mathrm{Yu}$ et al., who found an almost linear relation between diameter and wavelength of the first exciton, that is, $E_{1 \mathrm{st}} \approx D^{-1.0}$. [17] The dotted lines in Figure 4 are linear fits for each transition (except for those labelled by asterisks) at varying nanocrystal size and serve as a guide for the eye.

The trends of the size-dependent energies of the transitions in Figure 4 are clear. The energy for the first transition $(\alpha)$ increases linearly with $D^{-1.5}$ and extrapolates to $0.29 \mathrm{eV}$ for $D^{-1.5}=0$ (i.e., infinite crystal size), in excellent agreement with the room-temperature bandgap of bulk PbSe $(0.278 \mathrm{eV})$ at the L-point of the Brillouin zone 
(see Figure 1). The higher lying transitions extrapolate to approximately the same value and show a similar $D^{-1.5} \mathrm{de}$ pendence. Furthermore, the slopes of the linear fits (dotted lines) increase gradually for the higher lying transitions. These observations can be explained with a simple effective mass approximation as will be shown below. The above-described trend only holds up to transition $\zeta$. From transition $\eta$ and higher, the slopes of the linear fits abruptly change to a lower value, and the transitions do not extrapolate to $0.28 \mathrm{eV}$ for infinite crystal size. Transition $\eta$ extrapolates to a value of $1.57 \mathrm{eV}$ for $D^{-1.5}=0$, and the higher lying transitions $(\theta$ and $\iota)$ again increase in slope and also extrapolate to a value relatively close to $1.6 \mathrm{eV}$. We propose that transitions $\eta, \theta$, and $\iota$ in Figure 4 correspond to optical transitions at another point in the Brillouin zone of $\mathrm{PbSe}$ than the $\mathrm{L}$ point. In the band structure of bulk PbSe, there is a second bandgap at the $\Sigma$-point in the Brillouin zone with an energy of $1.60 \mathrm{eV}$, in excellent agreement with our experimental value of $1.57 \mathrm{eV}$ for the extrapolation of transition $\eta .^{[11,18]}$ We assign $\eta$ to a direct transition between a hole at the $\Sigma$ point and an electron at the $\Sigma$-point, probably having S-symmetry envelope functions. Transitions $\theta$ and $\iota$ are assigned to transitions between higher-energy envelope functions at the $\Sigma$-point. As can be seen from the multi-Gaussian fit in Figure $2 \mathrm{~A}$, transition $\eta$ has a large oscillator strength compared to the lower-energy transitions.

First, we will use effective mass theory to qualitatively explain the trends observed in Figure 4. As was mentioned above, the energies of transitions $\alpha$ to $\zeta$ increase (nearly) linearly with $D^{-1.5}$ and extrapolate to $0.28 \mathrm{eV}$, with an increasing slope for higher energy transitions. Effective mass theory yields the following expression ${ }^{[19-21]}$ for interband transitions with electrons and holes in the same orbitals (e.g. $\left.1 \mathrm{~S}_{\mathrm{h}} 1 \mathrm{~S}_{\mathrm{e}}, 1 \mathrm{P}_{\mathrm{h}} 1 \mathrm{P}_{\mathrm{e}}, \ldots\right)$ :

$E_{n l}^{\mathrm{opt}}(D)=E_{\mathrm{g}}+\frac{2 \chi_{n l}^{2} \hbar^{2}}{D^{2}}\left(\frac{1}{m_{\mathrm{e}}^{*}}+\frac{1}{m_{\mathrm{h}}^{*}}\right)-\frac{0.9 e^{2}}{\varepsilon_{\mathrm{in}} D}$

where $n l$ values are the quantum numbers of the electron and hole orbitals and $\chi_{n l}$ values are roots of the spherical Bessel function, which increase with increasing quantum number ${ }^{[19]} E_{\mathrm{g}}, \hbar, e$, and $\varepsilon_{\text {in }}$ correspond to the fundamental bandgap, Planck's constant, the electronic charge, and the dielectric constant inside the nanocrystal, respectively. Neglecting the last term (because of the high $\varepsilon_{\text {in }}$ of $\mathrm{PbSe}$ ), we find the following slope of the energy with respect to $D^{-2}$ :

$\mathrm{d} E / \mathrm{d}\left(D^{-2}\right) \propto \chi_{n l}^{2}\left(\frac{1}{m_{\mathrm{e}}^{*}}+\frac{1}{m_{\mathrm{h}}^{*}}\right)$

Corrections to the above expressions (e.g., the use of a finite potential barrier) lead to a weaker dependence of energy on diameter. ${ }^{[19]}$ However, it is clear that the slope of the curves in Figure 4 should increase with increasing quantum numbers of the orbitals involved, which is exactly what is observed. Furthermore, the slope of the different transitions is also determined by the effective masses of electrons and holes. The significantly smaller slope of transition $\eta$ compared to transition $\alpha$ in Figure 4 is an indication that the effective masses are larger at the $\Sigma$-point than at the Lpoint. Although the size dependence of the lowest energy transition is $E \approx D^{-1.5}$ (and not $D^{-2}$ ), we estimate the ratios of the effective masses at the L- and $\Sigma$-points from the slopes in Figure 4: $\left(1 / m_{\mathrm{e}}^{*}+1 / m_{\mathrm{h}}^{*}\right)_{\Sigma}=0.67\left(1 / m_{\mathrm{e}}^{*}+1 / m_{\mathrm{h}}^{*}\right)_{\mathrm{L}}$. If we assume electron-hole symmetry, we can deduce that $\left(m_{\mathrm{e}}^{*}\right)_{\Sigma}=\left(m_{\mathrm{h}}^{*}\right)_{\Sigma}=1.5\left(m_{\mathrm{e}}^{*}\right)_{\mathrm{L}}=0.15$. In view of the simplifying assumptions, this should be regarded as a rough estimate.

As was shown in Figure 3, tight-binding calculations can reproduce the experimental absorption spectrum of $\mathrm{PbSe}$ QDs with a diameter of $6.8 \mathrm{~nm}$ to a large extent. We have calculated from which point in the Brillouin zone the different transitions of the calculated spectrum originate. Figure 5 shows a 2D-contour plot of the projection of the QD wave functions on bulk states, weighted by the oscillator strength of the optical transitions within a certain energy range. The results are shown as a function of the wave vector $k$ of the bulk state in the (110) plane of the Brillouin zone, for both conduction and valence band states (upper and lower images, respectively). Figure 5A and $\mathrm{B}$ shows the distribution of all transitions within an energy range of 0.60 to $0.68 \mathrm{eV}$, corresponding to transition $\alpha$ in Figure 3. As expected, these transitions are centred exactly at the L-point in the Brillouin zone. Figure 5C and D show the oscillatorstrength-weighted distribution of all transitions within an energy range of 1.85 to $1.88 \mathrm{eV}$, corresponding to transition $\eta$ in Figure 3 (range indicated by the gray dashed vertical lines). Interestingly, these transitions have a clear $\Sigma$-character, confirming the above interpretation that transition $\eta$ originates from the $\Sigma$-point in the Brillouin zone. The tightbinding calculations find hundreds of different transitions (with $\Sigma$-character) around transition $\eta$, indicating a high density of states around the $\Sigma$-point in the Brillouin zone. This explains the large optical density around $1.85 \mathrm{eV}$ that is observed in both the calculated and experimental absorption spectra in Figure 3.

Furthermore, the size dependence of the energy of transitions $\alpha$ and $\eta$ was calculated using the same tight-binding approach. The results are included in Figure 4. As can be seen, the calculated size dependence of transition $\alpha$ nicely follows the experimentally obtained energies, although the dependence is not linear on this scale. The size dependence of the energy of transition $\eta$ is also reproduced by the tightbinding calculations. The effective masses of both electrons and holes can be extracted from the tight-binding calculations as well. The effective masses of the electrons (holes) are $0.167(0.135)$ in the $<110>$ direction, $-2.11(-1.53)$ in the $<001>$ direction, and $0.098(0.104)$ in the $<1 \overline{1} 0>$ direction. The negative effective mass in the $<001>$ direction results from the fact that the $\Sigma$-point is not a valley, but a saddle point. On average, this results in an effective mass of approximately 0.19 for electrons $\left(1 / m_{\mathrm{e}}^{*}=1 / 3^{*}\left(1 / m_{\mathrm{e},\langle 110\rangle}^{*}+1 / m_{\mathrm{e},\langle 001\rangle}^{*}+1 / m_{\mathrm{e},\{110\rangle}^{*}\right)\right.$ and 0.18 for holes (in a similar fashion) at the $\Sigma$-point in the Brillouin zone. This is in good agreement with the effective masses that were derived from the experimental data using effective mass theory.

MEG in PbSe nanocrystals through excitation of a highenergy photon has received considerable attention in the 

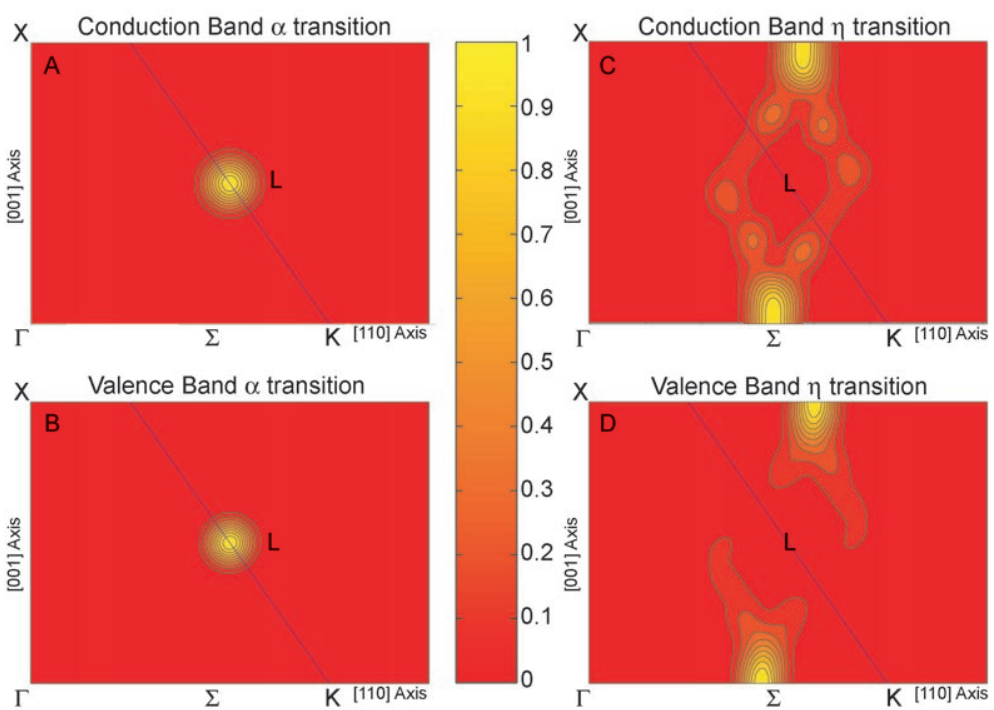

Figure 5. 2D-contour plot of the projection of the QD wave functions $\psi_{i}$ on bulk states $\phi_{k}$, weighted by the oscillator strength of the optical transitions $\psi_{i} \rightarrow \psi_{f}$ within a certain energy range $\left[\propto \sum_{i f}\left|\left\langle\psi_{i} \mid \psi_{f}\right\rangle\right|^{2}\left|\left\langle\psi_{i}|r| \psi_{f}\right\rangle\right|^{2}\right.$ with $\left.E_{\min }<E_{f}-E_{i}<E_{\max }\right]$. The results are shown as a function of the wave vector $k$ on the bulk state $\psi_{k}$ in the (110) plane of the Brillouin zone. (A) and (B) show all calculated transitions (for conduction and valence band states $\phi_{i}$, respectively) in an energy range of $E_{\min }=0.60 \mathrm{eV}$ to $E_{\max }=0.68 \mathrm{eV}$, corresponding to transition a in Figure 3. (C) and (D) show all calculated transitions in an energy range of $E_{\min }=1.85 \mathrm{eV}$ to $E_{\max }=1.88 \mathrm{eV}$, corresponding to transition $\eta$, as indicated by the gray dashed vertical lines lines in Figure 3.

past few years. ${ }^{[7-10]}$ The mechanism for MEG, however, is still under debate. Two theoretical papers used impact ionization (II) to explain the ultrafast carrier multiplication. ${ }^{[22,23]}$ In both cases, the II rate is calculated and compared to the rate of competing nonradiative decay mechanisms (i.e., phonon-assisted electron/hole cooling). Apart from II, a coherent superposition of multi-exciton and single-exciton states has also been proposed to explain MEG. ${ }^{[10,24]}$ In this case, the electron/hole cooling rate has to be slower than the multi-exciton thermalization rate and the rate of coupling between the single-and multi-exciton states. ${ }^{[24]}$ Finally, another group proposed the instantaneous formation of multi-excitons through weak coupling with a virtual singleexciton state to explain MEG, in which case the electron/ hole cooling does not play a role. ${ }^{[7]}$

The threshold for MEG in PbSe QDs of 6.8-nm diameter $\left(E_{\mathrm{g}}=0.64 \mathrm{eV}\right)$ was determined at $1.8 \mathrm{eV}{ }^{[9]}$ Here, we find that PbSe 6.8-nm QDs have transition $\eta$ at $1.9 \mathrm{eV}$. Similarly, Schaller et al. found that for smaller QDs $\left(E_{\mathrm{g}}=0.94 \mathrm{eV}\right)$, the threshold for MEG was at $2.8 \mathrm{eV}$ (ca. three times the bandgap) ${ }^{[8]}$ where we find transition $\eta$ at approximately two times the bandgap. However, Ellingson et al. found that for smaller PbSe QDs (3.9-5.4 nm, $E_{\mathrm{g}}$ from $\left.0.91-0.73 \mathrm{eV}\right)$, the threshold for MEG was between 2.1 and 2.9 times the bandgap, ${ }^{[10]}$ which is in better agreement with the position of transition $\eta$ that we find for these small nanocrystal sizes (between 2.2 and 2.6 times the bandgap). The observation that the experimentally obtained energy thresholds for MEG correspond with the energy of transition $\eta$ indicates that excitation into the $\eta$ state (at the $\Sigma$-point) may play a role in the mechanism of MEG. For example, electron/hole cooling to the L-valley may become significantly slower for an exciton at the $\Sigma$-point compared to an exciton within the L-valley. As was mentioned above, slow electron/hole cooling is crucial to explain efficient MEG by, for example, impact ionization.

\section{Conclusions}

In conclusion, we have measured and analyzed the optical absorption spectra of quasispherical PbSe nanocrystals ranging from 3.4 to $10.9 \mathrm{~nm}$ in diameter. We have identified, in total, 11 distinct optical transitions. Comparison with state-of-the-art calculated absorption spectra shows a good agreement for many of the features observed but also demonstrates that there are still important discrepancies between experiment and theory. By plotting all transition energies as a function of nanocrystal size, we find that the energy of the transitions changes as $E \propto D^{-1.5}$. Low-energy transitions extrapolate to $0.29 \mathrm{eV}$, in good agreement with the bandgap of bulk PbSe in the L-valley. High-energy transitions extrapolate to $1.57 \mathrm{eV}$, in good accordance with the bandgap of bulk PbSe at the $\Sigma$-point in the Brillouin zone. These higher-energy transitions are therefore ascribed to direct optical transitions at the $\Sigma$-point, and an estimate of the effective mass at this point of 0.15 for both electrons and holes is obtained. Tight-binding calculations confirm that the highenergy transitions originate from the $\Sigma$-point, and an average effective mass of $0.19(0.18)$ is calculated for electrons (holes), in good agreement with the experimentally derived value. It is shown that the reported threshold energy for multiple exciton generation in PbSe nanocrystals coincides with the energy of the first direct transition at the $\Sigma$-point, suggesting a relation between the two.

\section{Experimental Section}

The PbSe nanocrystals were synthesized inside an argon or nitrogen-purged glovebox. All chemicals were stored inside this glovebox. A stock solution of lead acetate trihydrate $(3.25 \mathrm{~g}$, Aldrich, $99.999 \%)$ in diphenyl ether $(10 \mathrm{~mL}$ DPE, Aldrich), oleic acid (7.5 mL, Aldrich, 90\%), and trioctyl phosphine (40 mL TOP, Fluka, $90 \%$ ) was prepared. The lead oleate precursor was prepared by heating the above 
mixture to over $70^{\circ} \mathrm{C}$ under vacuum $\left(<10^{-3} \mathrm{mbar}\right)$ for $1 \mathrm{~h}$ to remove trace amounts of water and acetate; a crucial step to obtain monodisperse spherical nanocrystals. ${ }^{[25]}$ The solution was allowed to cool to room temperature. Then $11.5 \mathrm{~mL}$ of this solution was mixed with a solution of selenium $(1.0 \mathrm{M}$, Alfa Aesar, $99.999 \%)$ in TOP $(1.7 \mathrm{~mL})$ and rapidly injected into DPE $(10 \mathrm{~mL})$ that was preheated to the desired injection temperature. In a typical synthesis, the injection temperature was $180^{\circ} \mathrm{C}$, the temperature dropped upon injection to $125^{\circ} \mathrm{C}$ and quickly reached the growth temperature of $135^{\circ} \mathrm{C}$. The size of the spherical nanocrystals was controlled by the growth time, which varied from $14 \mathrm{~s}$ ( $3.4 \mathrm{~nm} \mathrm{NCs)}$ to $30 \mathrm{~min}(10.9 \mathrm{~nm} \mathrm{NCs})$. The crude products were cleaned by the addition of a small volume of butanol (Aldrich, anhydrous, 99.8\%), centrifugation, and dissolution of the precipitate in tetrachloroethylene (Aldrich, anhydrous, $>99 \%$ ). This cleaning procedure was repeated once. Transmission electron microscopy images were obtained with a Philips Tecnai 12, a Fei Tecnai 10, and a Fei Tecnai 20 FEG instrument. Optical absorption spectra were recorded with a Perkin-Elmer Lambda 950 UV/Vis spectrophotometer. Additional absorption spectra and corresponding second-derivative spectra of PbSe QDs of different sizes can be found in the Supporting Information.

\section{Acknowledgements}

We thank B. Luigjes for preparing the PbSe nanocrystals, J. de Folter, S. Zevenhuizen, and H. Meeldijk for their assistance in obtaining TEM images. Financial support from the European Union network "FULLSPECTRUM" (SES6-CT-2003-502620) is gratefully acknowledged.

[1] J. M. An, A. Franceschetti, S. V. Dudiy, A. Zunger, Nano Lett. 2006, 6, 2728-2735.

[2] A. Sashchiuk, L. Amirav, M. Bashouti, M. Krueger, U. Sivan, E. Lifshitz, Nano Lett. 2004, 4, 159-165.
[3] C. B. Murray, S. H. Sun, W. Gaschler, H. Doyle, T. A. Betley, C. R. Kagan, IBM J. Res. Dev. 2001, 45, 47-56.

[4] F. W. Wise, Acc. Chem. Res. 2000, 33, 773-780.

[5] B. L. Wehrenberg, C. J. Wang, P. Guyot-Sionnest, J. Phys. Chem. B 2002, 106, 10634-10640.

[6] G. Konstantatos, I. Howard, A. Fischer, S. Hoogland, J. Clifford, E. Klem, L. Levina, E. H. Sargent, Nature 2006, 442, 180-183.

[7] R. D. Schaller, V. M. Agranovich, V. I. Klimov, Nat. Phys. 2005, 1, 189-194.

[8] R. D. Schaller, V. I. Klimov, Phys. Rev. Lett. 2004, 92, 186601.

[9] R. D. Schaller, M. Sykora, J. M. Pietryga, V. I. Klimov, Nano Lett. 2006, 6, 424-429.

[10] R. J. Ellingson, M. C. Beard, J. C. Johnson, P. R. Yu, O. I. Micic, A. J. Nozik, A. Shabaev, A. L. Efros, Nano Lett. 2005, 5, 865871.

[11] G. Allan, C. Delerue, Phys. Rev. B 2004, 70, 245321.

[12] I. Kang, F. W. Wise, J. Opt. Soc. Am. B 1997, 14, 1632-1646.

[13] P. Liljeroth, P. A. Z. van Emmichoven, S. G. Hickey, H. Weller, B. Grandidier, G. Allan, D. Vanmaekelbergh, Phys. Rev. Lett. 2005, 95, 086801.

[14] B. L. Wehrenberg, P. Guyot-Sionnest, J. Am. Chem. Soc. 2003, 125, 7806-7807.

[15] J. M. Pietryga, R. D. Schaller, D. Werder, M. H. Stewart, V.I. Klimov, J. A. Hollingsworth, J. Am. Chem. Soc. 2004, 126, $11752-11753$

[16] D. J. Norris, M. G. Bawendi, Phys. Rev. B 1996, 53, 16 33816346.

[17] W. W. Yu, J. C. Falkner, B. S. Shih, V. L. Colvin, Chem. Mater. 2004, 16, 3318-3322.

[18] E. A. Albanesi, C. M. I. Okoye, C. O. Rodriguez, E. L. P.Y. Blanca, A. G. Petukhov, Phys. Rev. B 2000, 61, 16 589-16 595.

[19] S. V. Gaponenko, Optical Properties of Semiconductor Nanocrystals, Cambridge University Press, Cambridge, UK 1998.

[20] A. Franceschetti, A. Williamson, A. Zunger, J. Phys. Chem. B 2000, 104, 3398-3401.

[21] L. Brus, J. Phys. Chem. 1986, 90, 2555-2560.

[22] A. Franceschetti, J. M. An, A. Zunger, Nano Lett. 2006, 6, 21912195.

[23] G. Allan, C. Delerue, Phys. Rev. B 2006, 73, 205423.

[24] A. Shabaev, A. L. Efros, A. J. Nozik, Nano Lett. 2006, 6, 28562863.

[25] A. J. Houtepen, R. Koole, D. L. Vanmaekelbergh, J. Meeldijk, S. G. Hickey, J. Am. Chem. Soc. 2006, 128, 6792-6793.

Received: August 2, 2007

Published online on December 20, 2007 